\title{
5. KnowledgExchange: a knowledge- brokering initiative in the Victorian child and family welfare sector
}

\author{
Cathy Humphreys and Richard Vines
}

\section{Introduction}

As the diameter of our knowledge increases, the circumference of our ignorance expands. (Anonymous stem-cell researcher) ${ }^{1}$

The words used by the anonymous stem-cell researcher could apply equally to the challenges of knowledge brokering in the children and families sector. Our knowledge base in the area is undoubtedly increasing; however, the ability to translate and use aspects of this knowledge base in practice in ways that make a difference to the lives of children and young people and their families remains a constant challenge.

This chapter initially describes a broad-based knowledge-brokering initiative in Victoria. It then concentrates on one aspect of this program: the way in which the information technology infrastructure for the sector and particularly the practices of managers and front-line workers using electronic data systems can be brokered to more usefully inform practice. It raises the question of whether these systems are barriers to or facilitators of good practice in the area. This is a question that has been addressed eloquently by Parton (2008), who questions whether social work and particularly child protection practice are being shifted from the 'terrain of the social' to the 'terrain of the informational'. This project used a knowledge broker (RV) to explore these changes in practice with frontline workers and managers.

While the primary goal of the project was to explore the role that an understanding of organisational data could play in supporting practice with vulnerable children and their families, it also raised the broader question of the interface between workers, managers, consumers and the technology of

1 We are grateful to Nick Collins, formerly with Glastonbury Child and Family Services, for making us aware of this quotation. 
electronic databases. The conceptual question is whether electronic databases are simply electronic versions of a paper file. This would suggest a first-order change in which there is merely the imposition of a different technology to support the same work with the goal (not always realised!) of an increase in efficiency (Ison and Russell 2000). Alternatively, the scale of technological advances could be such that a second-order change is occurring that shifts the nature of practice - as Parton (2008) suggests is now occurring. Second-order changes go beyond the question of efficiency ('how' to work better) to look at questions of purpose ('what' are we doing and 'why') (Checkland and Poulter 2006). The question then arises of how this shift can be used to enhance rather than undermine the interventions and the outcomes for vulnerable children and families. It is a critical question in which we feel a knowledge broker has a key role to play.

\section{The KnowledgExchange initiative}

Before focusing on the particular information technology project initiated by the knowledge broker, situating the work within its broader context provides an explanation for how this somewhat unusual knowledge-brokering project arose as part of a relationship between the Alfred Felton Chair in Child and Family Social Work and the Centre for Excellence in Child and Family Welfare.

The chair was established as a collaborative initiative between the Alfred Felton Trust, the Social Work Department at the University of Melbourne and the peak body for 95 member organisations in the child, youth and family services sector in Victoria. The role of the new chair was conceptualised as contributing to the knowledge base for the children and families sector in Victoria with a particular emphasis on capacity building in the community-sector organisations. Two aims were associated with this particular chair: the development of new and relevant research to inform practice and policy; and the implementation of current knowledge already developed, but under-utilised by the sector.

The second aim was supported by the employment for three years of a knowledge broker through a successful application by the centre to the Telstra Foundation. Work progressed to support a knowledge-brokering agenda for the centre and the chair in this collaborative initiative now branded 'KnowledgExchange'. The collaborative arrangement is depicted in Figure 5.1. Conceptions of the knowledge-brokering role itself have been influenced by the experience of those involved in the early stages of the project. For example, the knowledge broker was recruited from outside the community sector. He has contributed to an overall team approach that has shaped the project agenda by integrating perspectives derived from previous and practical experiences associated with 
knowledge management (Vines and Naismith 2002), including complex and technical matters associated with print and electronic text convergence (Vines 2006; Printing Industries Association of Australia 2004; RMIT 2004).

\section{Figure 5.1 Links between the Alfred Felton Research Program, the Centre for Excellence and the KnowledgExchange project}

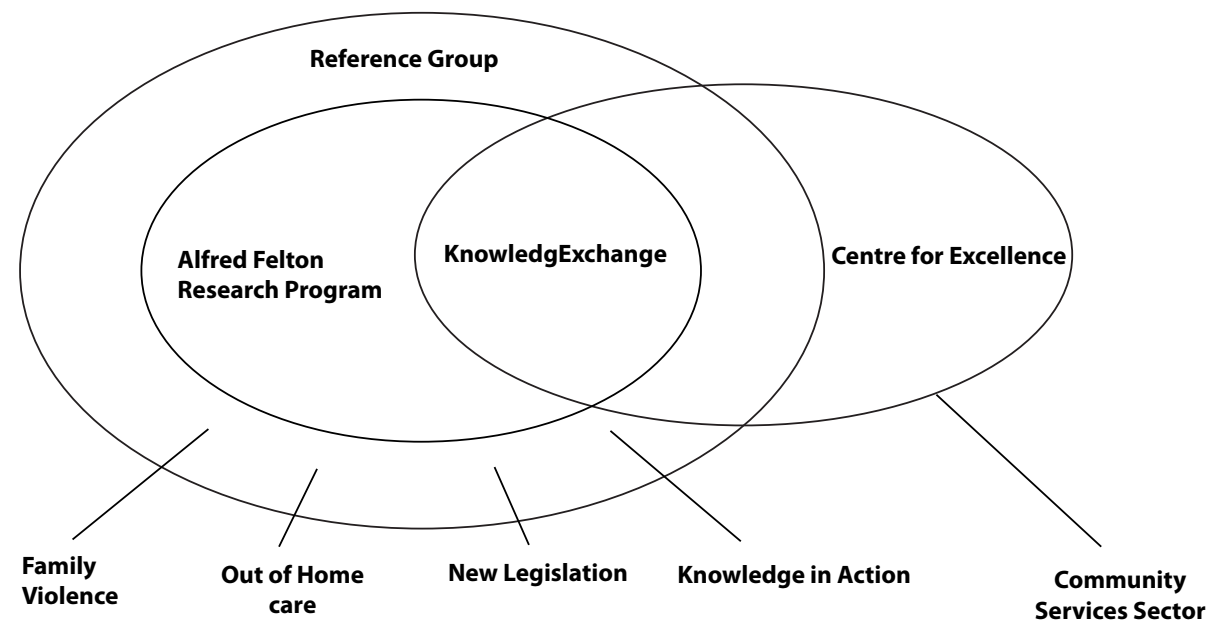

The Alfred Felton Research Program receives input from a reference group on four areas: family violence, out-of-home care, new legislation and knowledge in action.

Thanks to Jenny Higgins, the second knowledge broker for the KnowledgExchange project, for the figure.

At the heart of the KnowledgExchange agenda is the intention to impact on the lives of vulnerable children and their families primarily through the knowledge development of practitioners and their managers. The objective of the KnowledgExchange is to develop three key themes

- to deepen the culture of evidence-informed decision making within the child and family welfare sector

- to create an environment in which innovation can flourish within the existing networks of influence and activities being developed under the umbrella of the Centre for Excellence and the Alfred Felton Research Program headed by the chair

- to facilitate the sharing of practice wisdom and organisational knowledge within the children, youth and families sector in Victoria.

The project is assisted by the collaborative infrastructure provided by the two participating institutions. The centre provides advocacy, professional development, research, training, policy and program advice, publications and resources for the 95 participating community-sector organisations. The chair 
brings research and research utilisation expertise. Together, through a process of extensive consultation in the sector and with the help of a reference group of key stakeholders, three strands of work have been identified as priority areas. It is envisaged that each of the three strands will provide rich themes for the exploration of original research as well as the development of the KnowledgExchange project facilitated by the knowledge broker.

The first of the three strands of work is supporting earlier intervention with vulnerable children and their families through working with the Victorian family support initiative known as Child FIRST (which stands for Family Information, Referral and Support Team). This provides coordinated, community-based intake and family support to vulnerable children and families through a range of community-sector organisations. The initiative is supported by the Children, Youth and Families Act, 2005, which brings new ways of working into the family support-sector organisations. Two themes in this complex project have been highlighted

- The knowledge base for partnership working. New organisational forms of working are now emerging (and being imposed). The knowledge base to inform partnerships and network development is still embryonic, yet has a role to play in informing more efficient and effective forms of organisation for delivering services to children and their families.

- The use of data to inform and develop practice. This theme is progressively expanding to take in the broader facilitation of a strategic approach to community-sector organisation support systems infrastructure such as client information management, records management and web publishing. It is this aspect of the KnowledgExchange project that is the focus of the case study later in this chapter.

Family violence and its practice development within family support services is the second strand of work. In the past, intervention in this area has been provided primarily by family violence specialist workers. A new 'whole-ofgovernment' strategy for Victoria configures family services organisations, Child FIRST and a network of mainstream health and welfare organisations working together to deliver services. This requires significant new knowledge, particularly for those organisations in the children and families sector that have not traditionally engaged with this issue. Drawing this knowledge into family support services serves a range of agendas. One is developing a more informed workforce to intervene in the complex issues of family violence in collaboration with other key organisations.

The third strand of work-supporting quality and stability for children in outof-home care - is a particular priority and driver in the new children, youth 
and families legislation in Victoria. A number of projects are developing. For example, of relevance to this chapter has been the establishment of a seeding project funded through the University of Melbourne's 'Knowledge Transfer' program (University of Melbourne 2007). This initial and small-scale project has emerged through collaboration between the University's eScholarship Research Centre, the Alfred Felton Chair and the Centre for Excellence and forms part of a network of integrated knowledge-brokering initiatives. This particular intervention has been developed to bring to the surface the significant reform challenges associated with electronic data systems and other forms of record keeping for children and young people in out-of-home care. The 'Who Am I?' project has subsequently developed as an ARC Linkage project. This is providing a focus for consolidating the university's KnowledgExchange project and a range of other initiatives and aims to link past and present through highlighting the significance of record keeping and information management in the digital world. As part of this project, archivists and historians propose a new resource to support the accessibility of information about Victorian child welfare institutions and where record holdings are kept (<www.pathwaysvictoria. info $>$ ).

It is envisaged that the development of the KnowledgExchange project will underpin each of these strands of research, though the real configuration for each will take a different form, depending on the stakeholders involved in each project and the priorities they have in bridging the gaps between research, policy and practice. In this process, we have been assisted by the International Symposium in 2008 held in Dartington, England (<www.ripfa.org.uk $>$ ), which explored five different dimensions of knowledge-brokering work

- strategies for identifying evidence (the role of the literature review and meta-analysis)

- strategies for delivering evidence-informed practice

- supporting and exploring the role of in-house evaluation

- strategies for embedding evidence-informed practice

- strategies for evaluating the impact of a knowledge-brokering project (how do we know we are making a difference?).

Conceptually, the work draws on the cultures-in-context model of research use (Arney and Bromfield 2008). The model attempts to understand the different cultures of research, policy and practice within the context of wider domains of influence. Within this context, research and evidence are theorised as only two of many drivers that shape policy and practice. The knowledge broker sits within this context 'spanning' the different domains, scanning the emergent practices and policies and constructing opportunities to create strategies for the 
enhanced use of research in policy and practice. The five different dimensions of knowledge-brokering work provide the framework for or tool-kit of strategies from which the broker can draw in this 'spanning' work.

The project to explore the role of data systems in developing evidence-informed practice initially arose within the strand of 'early intervention'. It was here, in the new Child FIRST partnerships developed between different family service providers and statutory child protection (Victorian Department of Human Services), that the focus on the role of databases first arose as a 'live' issue for interrogation and exploration. The ramifications for out-of-home care and domestic and family violence also quickly emerged.

\section{Case study: exploring the use of data systems to develop evidence-informed practice}

It is unsurprising in the Victorian children, youth and families sector that interest in the electronic databases used by family services workers to support their practice can be galvanised. The 'Every Child Every Chance' reform in this sector, which resulted in a new systems configuration for child protection and family services, was driven partly by a careful analysis of the statutory child protection database in 2002. The analysis of these organisational data showed that: 60 per cent of notifications to child protection were re-notifications; an increasing number of notifications were closed with 'no further action' at intake; and the adult issues of domestic violence, mental illness and problematic substance use featured in more than 70 per cent of cases and yet the child protection system was not designed to respond to these complex issues. Moreover, it was predicted that within 10 years, one in five children in Victoria would come to the notice of this system, which was clearly failing to respond appropriately to their needs or to those of their parents and other family members (Allen Consulting 2003).

While other factors were also significant in driving the reform, the attention to evidence drawn from these administrative databases provides a continuing story that is well known within the Victorian Government and non-government sector. It provides a context in which the power of evidence to support reform has been demonstrated.

In spite of this history, however, the engagement between practitioners, managers and these organisational databases is complex. Consultation with front-line workers, community-sector organisation managers and researchers in the children and family services area by the chair and knowledge broker consistently raised the ubiquitous and contentious nature of the electronic data systems used by front-line workers. It also raised an interesting challenge for 
the definition of knowledge brokering given that the role of databases and data mining is generally conceptualised as part of a research agenda. Nevertheless, it is also a 'boundary-spanning' issue in that organisational data can provide direct knowledge to inform policy and practice. How databases are used, the opportunities and the limitations provided by the current databases and the impact on practice lent themselves to an aspect of KnowledgExchangenamely, the sharing of practice wisdom and organisational knowledge within the children, youth and families sector in Victoria. Generally, we would envisage attention to organisational databases and their use as fitting broadly within the strategies for delivering evidence-informed practice. The focus on the capacity to use data and information to monitor patterns at multiple levels within complex systems (including team, organisation, catchment, regional and state-wide levels) is, however, a particular subcategory of knowledge-brokering work.

A starting point for the initiative was created by a practice forum and a 'twilight seminar series' at which practitioners, managers and researchers were invited to present and discuss data issues that were impacting on practice. Slightly bemused practitioners, managers and academics wondered together about how the role of electronic databases could be such an engaging topic at five o'clock in the afternoon.

A number of issues emerged across the seminar series. It became clear that while electronic systems were accepted as a central part of human services practice, in their current form they were constantly problematised. The issues raised included

- the alienation of front-line workers from the data they input, which provide little feedback to them to inform their practice, and instead generally seem to be used by others

- the inadequacy of the designated fields (data elements) in the electronic data system, which might not reflect the context-specific work undertaken with the children and their families

- the lack of compliance by workers, which means that the data might not reflect their real work or the work of the organisation

- double handling of client information if paper-filing (analogue) systems are required in parallel with the electronic client information management (digital) systems, or where compliance requires logging information into two or more different electronic management systems

- the amount of time spent by workers at their computers, which, it is claimed, detracts from working with clients 
- the ethical issues raised by the automatic information sharing that can occur through the electronic data system - a particular issue when the statutory and non-government sectors share the same electronic data system

- the multiple electronic data systems used by any one organisation, which do not interface with each other and which do not allow the community-sector organisation to gain an overall picture of their work

- the sustainability of the electronic record into the future. A particular issue relates to those leaving care, who might want to access information about themselves and the organisational context in which they lived at a later date.

Presentations were not, however, entirely negative. An optimistic and progressive use for the state-wide databases lay in their potential to provide back to the sector meaningful reports about trends in family services practice at the state-wide and regional or catchment levels, as long as resources were provided for such analysis (Boffa 2007). Similarly, the creation of purpose-built databases was also providing meaningful data for some organisations, supporting their processes of referral and intake, as well as providing useful information on 'demand flow'.

In raising these issues in the seminar series, as well as at community forums and reference group meetings, a range of issues that coalesce around the information technology systems within each community-sector organisation has begun to be explored. A further step will now be taken with a one-day conference that will investigate the implications for community-sector organisations building their own custom-made systems, buying or sharing those developed by other organisations or relying on inputting into the government databases.

The next stages in this process of exploration are now occurring in an attempt to address some of the issues that had been problematised through the knowledge broker and practitioner-led seminar series. The successful research multi-agency application ('Who Am I?') to the ARC continues the exploration of the ways in which the electronic data are to be archived for those leaving care. A data-user group has also been established with the assistance of one of the presenters in the seminar series (Julie Boffa), a worker who spans social work and database analysis. Data reports arising from the network of Child FIRST consortia are being used to interpret and understand 'the flow of work' through networks in Victoria.

The establishment of this data-user group, which meets as part of the Child FIRST practitioner and manager forum, allows for analysis of quarterly reports as they become available. Aggregated reports from the Department of Human Services database are provided to the Child FIRST and family service alliances 
to show a state-wide overview. In addition, there are local government area and catchment-level reports, which provide the data at this level of aggregation allowing networks to understand the picture of work for their area.

The benefits for participants include

- the development of an understanding of the local and state patterns reported through an analysis of the data reports

- the ability to examine trends in data over time

- understanding both the strengths and limitations of the data, and how and whether they accurately reflect the practitioner and management experience of the work of the Child FIRST networks

- understanding about how the data can be fed back to managers and practitioners to inform the development of practice in Child FIRST networks.

We believe such an initiative has important potential. It can provide a catalyst for a more substantial partnership framework between the community-sector organisations and the Victorian Government. If such a collaborative mechanism can develop that results in the joint monitoring of emergent patterns of behaviour within the child and family welfare sector as a whole (Vines 2007), this could usher in a new approach to community capacity formation. It would also begin to address the processes through which front-line workers and managers are able to mine and analyse their own data to provide a more evidence-informed practice and an understanding of their place in patterns that emerge across the state.

The continuing exploration of the ways in which the information requirements of the sector and government are shifting and changing the nature of work with children and families creates another level of analysis for practitioners, managers and academics (Parton 2008). This is an intensely theoretical as well as practical issue, which points to the changing nature of our work in the 'electronic and digital era'. It creates a much-needed focus on the importance of information and communication technology systems as an integral part of creating linkages (or barriers) between policy personnel, practitioners and researchers. It is an issue that is both marginal (as a legitimate topic in children and families work) and central (as a time-consuming and mandated aspect of most children and families practice). 


\section{Conclusion}

The KnowledgExchange initiative provides opportunities to span the boundaries between different and often siloed cultures of research, practice and policy. In this process, academics, community-sector organisations and government workers are provided with opportunities to engage with knowledge sharing and problem solving. The project is informed by the 'cultures-in-context' model, which recognises that research and new knowledge are emergent and based on scanning for and creating opportunities for exchange within the worlds of research, policy and practice (Arney and Bromfield 2008). The initiative also recognises that an evidence-informed practice emerges when engaged practitioners and managers share their current practice in the context of an open inquiry in which research is one of several sources of new knowledge.

\section{References}

Allen Consulting 2003, Protecting Children: The child protection outcomes project, September, Department of Human Services, Government of Victoria, Melbourne.

Arney, F. and Bromfield, L. 2008, Strategies for delivering evidence informed practice: solutions not problems, Beyond the Rhetoric International Symposium, Dartington, UK, April 2008, viewed 3 May 2010, < http://www. aifs.gov.au/institute/pubs/papers/papers08.html>

Boffa, J. 2007, Advancing the Child FIRST reforms through meaningful data, Seminar Series: Data Systems to Support Evidence Informed Practice, University of Melbourne and Centre for Excellence in Child and Family Welfare, 28 June 2007.

Checkland, P. and Poulter, P. 2006, Learning for Action: A short definitive account of soft systems methodology and its use for practitioner, teachers and students, Wiley \& Sons, Chichester, UK.

Ison, R. and Russell, D. 2000, Agricultural Extension and Rural Development: Breaking out of knowledge transfer traditions, Cambridge University Press, UK.

Parton, N. 2008, 'Changes in the form of knowledge in social work: from the "social" to the "informational", British Journal of Social Work, vol. 38, pp. 253-269. 
Pathways 2009, Pathways web site, viewed 3 May 2010, <http://www. pathwaysvictoria.info/index.html>

Printing Industries Association of Australia 2004, Business Insights: Key learnings arising from EPICS projects, 2000-2004, Printing Industries Association of Australia, Newmarket, Qld, viewed 3 May 2010, <http://www.printnet. com.au/.../industry_reports_sub_pages/business_insights_key_learnings_ from_epics.html>

Royal Melbourne Institute of Technology (RMIT) 2004, The C-2-C Project: Creator to consumer in a digital age, Royal Melbourne Institute of Technology, Vic., viewed 27 March 2010, <http://c-2-cproject.com/background_html>

University of Melbourne 2007, Knowledge Transfer: Connecting Melbourne, University of Melbourne, Vic., p. 24, viewed 3 May 2010, http://web. archive.org/web/20080719030202/http://www.knowledgetransfer.unimelb. edu.au/KT_NOV07_webversion.pdf

Vines, R. 2006, From In-House Printing to Document Workflow. A guide for the better serving of readers, users and knowledge workers in an era of communications and digital media convergence, Canon Australia, North Ryde, NSW, viewed 3 May 2010, <http://www.nippa.com.au/pdf/RichardVinesJune06.pdf>

Vines, R. 2007, Towards a futures strategy for the Victorian community services sector: a knowledge perspective, VCOSS Congress Paper, August 2007, viewed 3 May 2010, <http:/www.vcoss.org.au/documents/VCOSS\%20docs/ Congress/2007/Presentations/B1 \%20VINES.pdf>

Vines, R. and Naismith, L. 2002, 'Exploring the foundations of knowledge management practice', in B. Cope and R. Freeman (eds), Developing Knowledge Workers in the Printing and Publishing Industries: Education, training and knowledge management in the publishing supply chain, from creator to consumer, Common Ground, Melbourne, viewed 3 May 2010, <http://richardvines. cgpublisher.com/product/pub.174/prod.1> 